\title{
COMMUNICATIONS
}

\section{Cis $\rightarrow$ trans conversion of formic acid by dissipative tunneling in solid rare gases: Influence of environment on the tunneling rate}

\author{
M. Pettersson ${ }^{\mathrm{a})}$ \\ Laboratory of Physical Chemistry, P.O. Box 55, FIN-00014, University of Helsinki, Finland \\ E. M. S. Maçôas \\ Laboratory of Physical Chemistry, P.O. Box 55, FIN-00014, University of Helsinki, Finland \\ and Department of Chemistry-CQC, University of Coimbra, P-3004-535, Coimbra, Portugal \\ L. Khriachtchev and J. Lundell \\ Laboratory of Physical Chemistry, P.O. Box 55, FIN-00014, University of Helsinki, Finland \\ R. Fausto \\ Department of Chemistry-CQC, University of Coimbra, P-3004-535, Coimbra, Portugal \\ M. Räsänen \\ Laboratory of Physical Chemistry, P.O. Box 55, FIN-00014, University of Helsinki, Finland
}

(Received 21 August 2002; accepted 23 September 2002)

The relaxation of the higher-energy cis conformer of formic acid to the lower-energy trans form by a tunneling mechanism has been investigated in low-temperature rare gas matrices. In the temperature range $8-60 \mathrm{~K}$, the tunneling takes place dominantly from the vibrational ground state of the cis form and the temperature dependence of the tunneling rate constant is influenced by the interactions with the environment. The temperature-dependent tunneling rates for $\mathrm{HCOOH}$ and $\mathrm{DCOOH}$ in solid $\mathrm{Ar}, \mathrm{Kr}$, and $\mathrm{Xe}$ are measured including data for molecules in different local environments within each host. It was found that the medium and the local environment has a significant influence on the tunneling rate. (C) 2002 American Institute of Physics.

[DOI: $10.1063 / 1.1521429]$

In reaction kinetics, tunneling of atoms is often negligible compared with over-barrier transitions. At very low temperatures, however, the population of energy states above the barrier becomes exceedingly small and tunneling becomes comparatively more important. ${ }^{1}$ In a condensed environment, phonons participate in a tunneling reaction and the environment should have some effect on tunneling reactions. ${ }^{2,3}$ However, in several previous experiments it was found that the tunneling rate constant was unaffected by the change of solvent. ${ }^{1,4-6}$ In this work, we have studied the conversion of cis formic acid ( $\mathrm{HCOOH})$ to trans formic acid in solid rare gases $(\mathrm{Ar}, \mathrm{Kr}, \mathrm{Xe})$. This reaction is dominated by tunneling from the vibrational ground state at temperatures below $60 \mathrm{~K}$. The results show that the tunneling rate depends strongly on the environment.

The samples were made by mixing vapors of formic acid (FA) (KEBO lab, >99\%) or its isotopomers (IT Isotope 95\%-98\% deuteration) with rare gases ( $\mathrm{Rg}$ ) Ar (AGA, 99.9999\%), Kr (Air Liquid, 99.95\%), Xe (AGA, 99.997\%) in the gas phase in a proportion $\mathrm{FA} / \mathrm{Rg} \approx 1 / 1000$. The gas mixture was deposited on a CsI substrate at $15 \mathrm{~K}(\mathrm{Ar}), 25 \mathrm{~K}(\mathrm{Kr})$ or $35 \mathrm{~K}(\mathrm{Xe})$ yielding highly monomeric matrices with respect to FA. Thickness of the sample was typically about 100 $\mu \mathrm{m}$. After deposition, the samples were cooled to $\sim 8 \mathrm{~K}$

${ }^{\text {a)} E l e c t r o n i c ~ a d d r e s s: ~ p e t t e r s @ c s c . f i ~}$ which was the lower limit for the cryostat (APD DE 202 A). The spectra were measured with a FTIR spectrometer (Nicolet $60 \mathrm{SX}$ ) with a resolution of 1 or $0.25 \mathrm{~cm}^{-1}$.

FA has energy minima in two planar forms differing by orientation of the hydroxyl group as shown in Fig. 1. The interconversion of the conformers involves mainly the torsional motion of the hydroxyl group. In the gas phase, cis-FA is $1365 \pm 30 \mathrm{~cm}^{-1}$ higher in energy than trans-FA. ${ }^{7}$ The barrier from trans to cis has been calculated to be $\sim 4200$ $\mathrm{cm}^{-1 .}$. In this work, cis-FA was prepared by exciting the vibrational transitions of trans-FA in rare-gas matrices with narrowband infrared radiation of an optical parametric oscillator (Sunlite, Continuum, FWHM $\sim 0.1 \mathrm{~cm}^{-1}$ ). The excitation energy flows into the torsional coordinate inducing the conformer conversion. ${ }^{9}$ The IR spectra of cis and trans FA differ significantly from each other making it possible to distinguish them easily in rare-gas matrices. ${ }^{9} \mathrm{FA}$ is trapped in several sites corresponding to different local environments and the corresponding IR absorption frequencies differ typically a few $\mathrm{cm}^{-1}$ as illustrated in Fig. 2 for cis-FA in solid Xe. Cis-FA was generated site-selectively by irradiating molecules in a separate site at a time similarly to our matrixisolation studies on HONO. ${ }^{10}$ In this way, the tunneling kinetics of FA in different sites could be investigated. There was no interconversion between different site groups either in IR pumping or in relaxation by tunneling. 


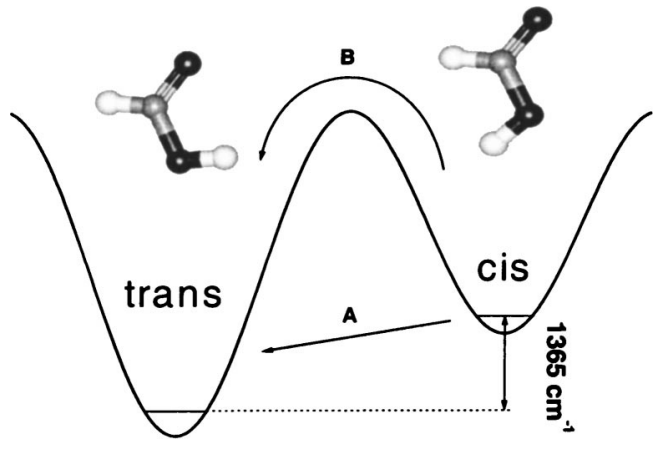

FIG. 1. Cis and trans formic acid and the torsional potential which connects the two conformers. The energy difference between the conformers is the experimental gas phase result from the Ref. 7. The arrows represent the conformer conversion processes by (A) tunneling and (B) over-barrier reactions.

Once formed, cis-FA converts back to trans-FA in a timescale of minutes even below $20 \mathrm{~K} .{ }^{9}$ At these temperatures, this reaction occurs purely via tunneling. Indeed, according to the $a b$ initio calculations, the barrier $\left(E_{a}\right)$ for the cis $\rightarrow$ trans isomerization is $\sim 2800 \mathrm{~cm}^{-1,8}$, which gives, according to the Arrhenius formula $k=A^{*} \exp \left(-E_{a} / k T\right)$ and using the torsional frequency for the pre-exponential factor $A$, a rate constant of $\sim 10^{-16} \mathrm{~s}^{-1}$ even at $60 \mathrm{~K}$. This rate is more than 13 orders of magnitude smaller than what is observed in our experiments. The over-barrier reaction should become significant at temperatures only above $\sim 110 \mathrm{~K}$. The torsional mode, which mainly corresponds to the tunneling coordinate, is the lowest frequency vibration in cis-FA at $\sim 500 \mathrm{~cm}^{-1}$. 9 It follows that, at the highest temperatures of our experiments $(60 \mathrm{~K})$ the population of the first excited torsional level is $<10^{-5}$. We have estimated by using the WKB approximation ${ }^{1}$ for the penetration probability and ab initio calculated (MP2/6-311++G(2d,2p), GAUSSIAN 98) ${ }^{11}$ torsional potential that the permeability of the barrier from the first excited torsional level is $\sim 4$ orders of magnitude higher than from the ground state. Therefore, at low temperatures the tunneling reaction takes place essentially from the ground vibrational state of cis-FA and the tunneling from the thermally excited vibrational states may become important only at temperatures above $60 \mathrm{~K}$. In this respect, FA is an

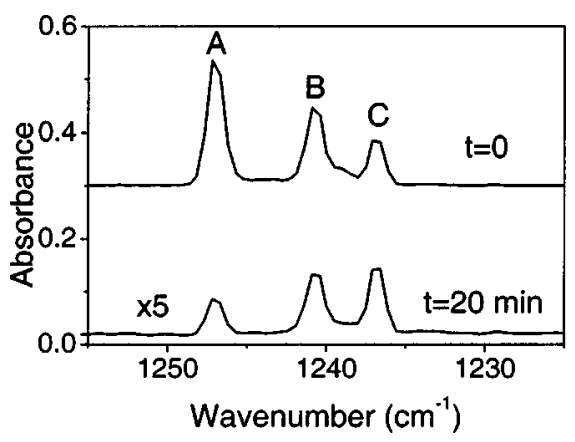

FIG. 2. IR absorption of cis- $\mathrm{HCOOH}$ in the $\mathrm{CO}-\mathrm{COH}$ deformation fundamental region in solid $\mathrm{Xe}$ at $8 \mathrm{~K}$. A, B, and $\mathrm{C}$ denote the absorptions corresponding to various sites (local environment). The lower trace was measured 20 min after the upper trace showing the different tunneling rates for the three sites.
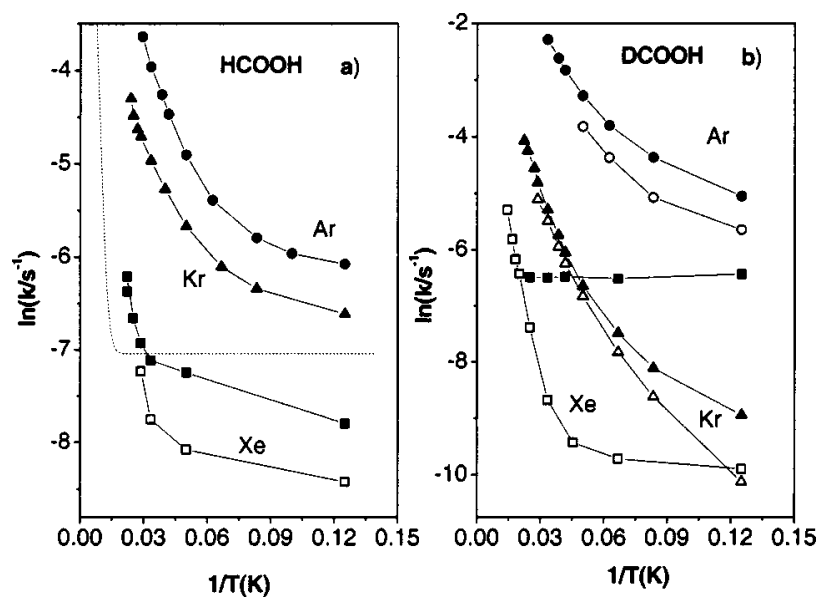

FIG. 3. Arrhenius plots for tunneling of (a) $\mathrm{HCOOH}$ and (b) $\mathrm{DCOOH}$ in various matrices. Data for two representative sites for each host are shown as solid and open symbols. The data for two sites shown in plot (a) for Xe as solid and open squares correspond to labels A and B in Fig. 2, respectively. For comparison, a simulated temperature dependence for tunneling from two lowest intramolecular vibrational states is shown as a dotted line.

ideal system to study the effect of the environment on tunneling.

The kinetics of the tunneling reaction was measured by FTIR spectroscopy after the trans $\rightarrow$ cis IR pumping was stopped by following the decrease in intensity of the $\mathrm{CO}-$ COH deformation absorption band of cis FA at $\sim 1250 \mathrm{~cm}^{-1}$ (see Fig. 2). As an additional complication, it was found that the spectrometer glowbar radiation increases the rate of the cis $\rightarrow$ trans conversion significantly. By using an interference filter that blocks the radiation above $\sim 1500 \mathrm{~cm}^{-1}$, the effect of glowbar was suppressed and tunneling rates could be reliably measured. It was also estimated that the room temperature blackbody radiation from the cryostat windows did not affect the results. The Arrhenius plots for $\mathrm{HCOOH}$ and $\mathrm{DCOOH}$ in various rare-gas solids including representative examples of different sites (indicated by solid and open symbols) are presented in Fig. 3. The isomerization rate for HCOOD was also measured in $\mathrm{Ar}$ and it was at least three orders of magnitude smaller at $8 \mathrm{~K}$ than for $\mathrm{HCOOH}$.

The major conclusion drawn from Fig. 3 is that the tunneling rate depends crucially on the solid host and in most cases also on the local matrix morphology (matrix-site effect). The temperature dependencies are also in definite contrast with the behavior that tunneling from the thermally excited intramolecular vibrational levels would show. To make this clear we show in Fig. 3(a) by a dotted line the simulated temperature dependence assuming tunneling from the two lowest torsional levels and four orders of magnitude higher tunneling probability from the first excited level than from the ground state. Clearly, comparing this result with the experiments suggests that the experimental temperature dependencies originate from the interaction of FA with the environment. For $\mathrm{HCOOH}$, the tunneling rate decreases from $\mathrm{Ar}$ to Xe by one order of magnitude at $8 \mathrm{~K}$. The rates for the two representative sites in solid Xe differ by a factor of $\sim 2$ at 8 $\mathrm{K}$ (see also Fig. 2). In $\mathrm{Ar}$ and $\mathrm{Kr}$ matrices the molecules in various sites have the same rates within the experimental error. For DCOOH, the different sites show different tunnel- 
ing rates in all the hosts. The tunneling rate for $\mathrm{DCOOH}$ in $\mathrm{Ar}$ is more than two orders of magnitude higher than in $\mathrm{Kr}$ at $8 \mathrm{~K}$. The rates for molecules in different sites in Xe differ by a factor of $\sim 30$ at $8 \mathrm{~K}$. It is also interesting to compare the tunneling rates of $\mathrm{HCOOH}$ and $\mathrm{DCOOH}$ in the same host. It is seen that the tunneling rates change considerably despite the deuteration of the carbon bound hydrogen shifts down the torsional frequency less than $2 \%$. In solid Ar at $8 \mathrm{~K}$, DCOOH tunnels three times faster than $\mathrm{HCOOH}$. In $\mathrm{Kr}$ matrices, the trend is opposite: $\mathrm{HCOOH}$ tunnels $\sim 30$ times faster than $\mathrm{DCOOH}$. In Xe host, the difference in rate constants depends strongly on which sites are compared.

These observations demonstrate that both changing the solid host, and changing the local environment within the same host can influence strongly the tunneling rates, the differences in the rate constants exceeding two orders of magnitude at $8 \mathrm{~K}$. Related phenomena have been reported for reorientation of $\mathrm{NH}_{3} \mathrm{D}^{+}$ions in Tutton salts. ${ }^{12}$ In those experiments, however, the tunneling system was defined by the environment (rotation of $\mathrm{NH}_{3} \mathrm{D}^{+}$between the inequivalent sites). In the present case, the tunneling system (internal rotation of formic acid) is well defined without the environment and therefore the effect of environment on the tunneling reaction is probed.

Next, we try to qualitatively understand different factors influencing the tunneling rate. Due to the large asymmetry of the torsion potential (see Fig. 1) the tunneling process involves excited vibrational states of trans-FA. In this respect, the energy difference between the cis and trans isomers and the vibrational level structure of trans are important to consider. It should be noticed that the two isomers have markedly different dipole moments $(3.79 \mathrm{D}$ for cis; $1.42 \mathrm{D}$ for trans $)^{7}$ and hence their solvation energies are different. We estimated the solvation energies of trans and cis-FA in various hosts within the frame of the polarized continuum (PCM) model in GAUSSIAN 98 (Ref. 11) at the MP2/aug-ccpvTZ level using the calculated gas phase structures and the dielectric constants of the rare gases [1.63 (Ar); $1.88(\mathrm{Kr})$; $2.19(\mathrm{Xe})] .^{13}$ The differences for solvation energies of the $c i s$ and trans forms are $319 \mathrm{~cm}^{-1}(\mathrm{Ar}), 412 \mathrm{~cm}^{-1}(\mathrm{Kr})$, and 508 $\mathrm{cm}^{-1}(\mathrm{Xe})$ indicating that solvation in a dielectric medium has a significant effect on the energetics. According to similar calculations performed on the transition state, the cis $\rightarrow$ trans barrier increases from Ar to $\mathrm{Kr}$ by $43 \mathrm{~cm}^{-1}$ and from $\mathrm{Kr}$ to $\mathrm{Xe}$ by $44 \mathrm{~cm}^{-1}$. The vibrational energy level structure of trans FA relative to the ground state of cis-FA is shown in Fig. 4 for $\mathrm{HCOOH} / \mathrm{Ar}, \mathrm{DCOOH} / \mathrm{Ar}$ and $\mathrm{HCOOH} /$ $\mathrm{Xe}$. The position of the ground state of cis-FA estimated by the solvation calculations is shown with a dashed line in Fig. 4. Due to the limited accuracy of the solvation model the position of the ground state of cis-FA should be considered qualitative. Therefore, the discussion below is mostly hypothetical with respect to the exact energetics. In solid Ar, the smallest energy gap for the relaxation from the ground state of $c i s-\mathrm{HCOOH}$ is connected with the first excited state of the $\nu_{8}$ mode ( $\mathrm{C}-\mathrm{H}$ out of plane wag) of the trans form. In contrast, in solid Xe, solvation lowers the energy of cis-FA and the most energetically favorable relaxation channel occurs to the first excited states of $\nu_{7}$ (OCO scissors) and $\nu_{9}$ (torsion)

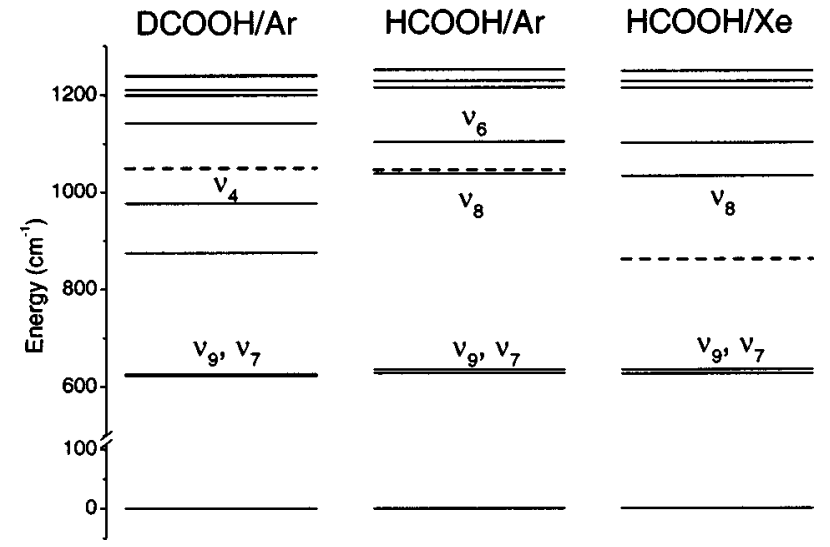

FIG. 4. Vibrational level structure of trans $\mathrm{HCOOH}$ and trans $\mathrm{DCOOH}$ as determined from their infrared spectra. The energy of the ground state of cis FA estimated by using the gas-phase energetics (Ref. 7) and $a b$ initio calculated solvation energies for the two conformers is shown with a dashed line. The labels for selected vibrational states correspond to torsion $\left(\nu_{9}\right)$, OCO scissor $\left(\nu_{7}\right), \mathrm{C}-\mathrm{H}$ out-of-plane wag $\left(\nu_{8}\right), \mathrm{C}-\mathrm{D}$ rocking $\left(\nu_{4}\right)$, and $\mathrm{COH}-\mathrm{CO}$ deformation $\left(\nu_{6}\right)$ modes (Ref. 9$)$.

modes. In solid Ar, one-phonon emission is sufficient to dissipate the excess energy (Debye frequency of $\mathrm{Ar}=93 \mathrm{~cm}^{-1}$ ) while in $\mathrm{Xe}$, a higher-order multiphonon process is required (Debye frequency of $\mathrm{Xe}=64 \mathrm{~cm}^{-1}$ ). ${ }^{14}$ We can conclude from these examples that at least three factors contribute to the observed differences in the tunneling rates: (1) Change in the barrier height due to solvation which, according to the calculations, is relatively small in this case. (2) Due to differences in solvation energies, in different environments phonon-assisted tunneling may occur between different levels. The coupling between vibrational levels can vary strongly and therefore the effect on the tunneling rate can be large. (3) Change of the magnitude of the energy gap between the tunneling levels changes the order of the phonon process providing the energy dissipation. The differences of the rates between $\mathrm{HCOOH}$ and $\mathrm{DCOOH}$ should mainly originate from the change of the intrinsic level structure upon deuteration (see Fig. 4). The site effect can be explained on the same basis: It is plausible to assume that molecules in various sites have different solvation energies because the cavity size and geometry are key factors in the solvation. Additionally, coupling with phonons can be different for various sites.

The temperature dependencies of the rate constants are roughly described as $k(T) \propto T^{3}$ or $T^{4}$. Previously, roughly $T^{4}$ dependence was observed for translational tunneling of the acidic protons in benzoic acid dimers. ${ }^{15}$ Theoretically, it was shown that $T^{2}$ or $T^{3}$ dependencies may arise from twophonon emission/absorption and Raman processes. ${ }^{16,17}$ Recently, a much stronger temperature dependence was reported for the $\mathrm{D}+\mathrm{HD} \rightarrow \mathrm{D}_{2}+\mathrm{H}$ reaction in solid HD (Ref. 18) and the origin of the effect was considered theoretically. ${ }^{3,19}$ The present observations should be valuable in testing theories for the temperature dependence of dissipative tunneling. In addition to the fundamental value the observed phenomenon can be used for solid-phase stabilization of desired species affected by tunneling decay by choosing a suitable environment. ${ }^{20}$ It is interesting to note that 
indirect evidence of the influence of environment on a cistrans isomerization tunneling reaction of hydroquinone in $\mathrm{Ar}$ and Xe matrices has been recently reported. ${ }^{21}$

In summary, it has been shown here that despite the relatively high energy barrier $\left(\sim 2800 \mathrm{~cm}^{-1}\right)$ cis-FA converts to trans-FA in timescale of minutes even at $8 \mathrm{~K}$ via pure tunneling mechanism. The tunneling rate depends strongly on the solid host and even on the local environment within a particular host, the differences in the tunneling rate constants exceeding two orders of magnitude at $8 \mathrm{~K}$.

This work was supported by the Academy of Finland. One of the authors (E.M.S.M.) acknowledges a Ph.D. student grant of the Portuguese Foundation for Science and Technology.

${ }^{1}$ R. P. Bell, in The Tunnel Effect in Chemistry (Chapman and Hall, London, 1980).

${ }^{2}$ V. A. Benderskii, D. E. Makarov, and C. A. Wight, in Chemical Dynamics at Low Temperatures (Wiley, New York, 1994).

${ }^{3}$ G. K. Ivanov, M. A. Kozhusner, and L. I. J. Trakhtenberg, Chem. Phys. 113, 1992 (2000).

${ }^{4}$ W. Al-Soufi, K. H. Grellmann, and B. Nickel, J. Phys. Chem. 95, 10503 (1991).

${ }^{5}$ J. Hennig and H.-H. Limbach, J. Chem. Soc., Faraday Trans. 2 75, 752 (1979).
${ }^{6}$ G. Brunton, J. A. Gray, D. Griller, L. R. C. Barclay, and K. U. Ingold, J. Am. Chem. Soc. 100, 4197 (1978).

${ }^{7}$ W. H. Z. Hocking, Z. Naturforsch. A 31A, 1113 (1976).

${ }^{8}$ J. D. Goddard, Y. Yamaguchi, and H. F. Schaefer III, J. Chem. Phys. 96, 1158 (1992).

${ }^{9}$ M. Pettersson, J. Lundell, L. Khriachtchev, and M. Räsänen, J. Am. Chem. Soc. 119, 11715 (1997).

${ }^{10}$ L. Khriachtchev, J. Lundell, E. Isoniemi, and M. Räsänen, J. Chem. Phys. 113, 4265 (2000).

${ }^{11}$ M. J. Frisch, G. W. Trucks, H. B. Schlegel et al., GAussian 98, Revision A.9, Gaussian, Inc., Pittsburgh, PA, 1998.

${ }^{12}$ H. L. Strauss, Acc. Chem. Res. 30, 37 (1997).

${ }^{13}$ H. E. Hallam and G. F. Scrimshaw, in Vibrational Spectroscopy of Trapped Species, edited by H. E. Hallam (Wiley, London, 1973).

${ }^{14} \mathrm{H}$. J. Jodl, in Chemistry and Physics of Matrix-Isolated Species, edited by L. Andrews and M. Moskovits (North-Holland, Amsterdam, 1989).

${ }^{15}$ C. Rambaud, A. Oppenländer, M. Pierre, H.-P. Tromsdorff, and J.-C. Vial, Chem. Phys. 136, 335 (1989).

${ }^{16}$ R. Silbey and H.-P. Trommsdorff, Chem. Phys. Lett. 165, 540 (1990).

${ }^{17}$ A. Suárez and R. J. Silbey, Chem. Phys. 94, 4809 (1991).

${ }^{18}$ T. Kumada, K. Komaguchi, Y. Aratono, and T. Miyazaki, Chem. Phys. Lett. 261, 463 (1996).

${ }^{19}$ M. V. Basilevsky and G. V. Davidovitch, J. Chem. Phys. 115, 6083 (2001).

${ }^{20}$ L. Khriachtchev, E. Maçôas, M. Pettersson, and M. Räsänen, J. Am. Chem. Soc. 124, 10994 (2002).

${ }^{21}$ A. Nobuyuki, S. Kudoh, M. Takayanagi, and M. Nakata, Chem. Phys. Lett. 356, 133 (2002). 
The Journal of Chemical Physics is copyrighted by the American Institute of Physics (AIP). Redistribution of journal material is subject to the AIP online journal license and/or AIP copyright. For more information, see http:/ojps.aip.org/jcpo/jcpcr/jsp Copyright of Journal of Chemical Physics is the property of American Institute of Physics and its content may not be copied or emailed to multiple sites or posted to a listserv without the copyright holder's express written permission. However, users may print, download, or email articles for individual use. 
The Journal of Chemical Physics is copyrighted by the American Institute of Physics (AIP). Redistribution of journal material is subject to the AIP online journal license and/or AIP copyright. For more information, see http://ojps.aip.org/jcpo/jcper/jsp 